\title{
Ginsparg-Wilson fermion propagators and chiral condensate
}

\author{
Ting-Wai Chiu* \\ Department of Physics, National Taiwan University, Taipei, Taiwan 106, Republic of China
}

(Received 29 October 1998; published 29 June 1999)

\begin{abstract}
By exploiting chiral symmetry, we derive analytic formulas for the massless and the massive GinspargWilson fermion propagators. Using these formulas, we derive an expression for the chiral condensate which is the order parameter for spontaneous chiral symmetry breaking in QCD. These formulas provide the proper way to compute the fermion propagators and may save a significant amount of computing time especially for large lattices in four dimensions. [S0556-2821(99)00415-4]
\end{abstract}

PACS number(s): 11.15.Ha, 11.30.Fs, 11.30.Rd

It is well understood that the proper way to break the chiral symmetry of a chirally symmetric Dirac operator $D_{c}$ on the lattice is to replace $D_{c}$ by

$$
D=D_{c}\left(\mathbb{I}+R D_{c}\right)^{-1},
$$

where $R$ is any Hermitian operator which is local in position space and trivial in Dirac space. Then the Dirac operator $D$ satisfies the Ginsparg-Wilson (GW) relation [1]

$$
D \gamma_{5}+\gamma_{5} D=2 D \gamma_{5} R D
$$

where the particular form of the chiral symmetry breaking on the right-hand side (RHS) having one $\gamma_{5}$ sandwiched by two Dirac operators is the signature of the Ginsparg-Wilson fermion. This implies chiral Ward identities, nonrenormalization of vector and flavor nonsinglet axial vector currents, and the nonmixing of operators in different chiral representations, as shown by Hasenfratz [2]. The general solution [3,4] of the GW relation, Eq. (1), can be regarded as a chiral symmetry breaking transformation which gaurantees that a zero mode of $D_{c}$ is also a zero mode of $D$ and vice versa; hence the index of $D$ is equal to the index of $D_{c}$. Then it is obvious to see that the presence of $R$ cannot produce a zero mode for $D$ if $D_{c}$ does not possess any zero modes in topologically nontrivial background gauge fields. We refer to Refs. [4,5] for further discussions on topological characteristics of $D\left(D_{c}\right)$. Now we can replace $R$ in Eq. (2) by any two Hermitian operators $S$ and $T$ having the same properties of $R$, and rewrite Eq. (2) as

$$
D \gamma_{5}(\mathbb{I}-S D)+(\mathbb{I}-D T) \gamma_{5} D=0
$$

then it is evident that the action $A=\bar{\psi} D \psi$ is invariant under the finite chiral transformation on the lattice:

$$
\begin{aligned}
& \bar{\psi} \rightarrow \bar{\psi} \exp \left[i \theta(\mathbb{I}-D T) \gamma_{5}\right], \\
& \psi \rightarrow \exp \left[i \theta \gamma_{5}(\mathbb{I}-S D)\right] \psi,
\end{aligned}
$$

where $\theta$ is a global parameter. This generalizes the infinitesimal chiral transformation observed by Lüscher [6]. In general, we can regard the Ginsparg-Wilson relation in the form

\footnotetext{
*Email address: twchiu@phys.ntu.edu.tw
}

of Eq. (3) as the exact chiral symmetry on the lattice, which recovers the usual chiral symmetry $\gamma_{5} D+D \gamma_{5}=0$ in the continuum limit $(a \rightarrow 0)$. Such a generalization seems to be cruical for formulating chiral gauge theories on the lattice.

In this paper, we will concentrate on the problem of evaluating the fermion propagator $D^{-1}$ of the GinspargWilson fermion. From Eq. (1), we immediately obtain

$$
D^{-1}=D_{c}^{-1}+R
$$

Hence, the fermion propagator $D^{-1}$ is completely determined for any $R$ if the chiral fermion propagator $D_{c}^{-1}$ has been evaluated. In fact, the long distance properties of $D^{-1}$ are governed by the long distance properties of $D_{c}^{-1}$ since $R$ is supposed to be local. Furthermore, we will show that the short distance behavior of $D^{-1}$ which is relevant to the chiral condensate is also determined by $D_{c}^{-1}$. Therefore the physics of any Ginsparg-Wilson fermion operator $D$ actually lies in its chiral limit $D_{c}$. However, only $D_{c}$ itself is not sufficent to constitute the correct lattice fermion operator. According to the Nielson-Ninomiya no-go theorem [7], if we require $D$ to be local and free of species doubling, then chiral symmetry $\left\{\gamma_{5}, D\right\}=0$ must be broken. The transformation in Eq. (1) provides the proper chiral symmetry breaking which not only preserves the correct physics of $D_{c}$, but also removes its nonlocality for any gauge configurations as well as its singularities in topologically nontrivial gauge fields. We note in passing that the locality requirement for the $\mathrm{GW}$ fermion should be imposed on the unitary operator $V$ in Eq. (8) rather than on $D$, since the nonlocality of $V$ is due to the initial construction and thus cannot be diminished, while the locality of $D$ is always gauranteed by a properly chosen $R$ in Eq. (1).

In the following, we derive a formula for the chiral fermion propagator for any $D_{c}$ satisfying the Hermiticity condition

$$
\gamma_{5} D_{c} \gamma_{5}=D_{c}^{\dagger}
$$

The Hermiticity condition and the chiral symmetry of $D_{c}$ imply that $D_{c}$ is anti-Hermitian; thus there exists a one-toone correspondence between $D_{c}$ and a unitary operator $V$,

$$
D_{c}=(\mathbb{I}+V)(\mathbb{I}-V)^{-1}, \quad V=\left(D_{c}-\mathbb{I}\right)\left(D_{c}+I\right)^{-1},
$$


where $V$ also satisfies the Hermiticity condition $\left(\gamma_{5} V \gamma_{5}\right.$ $=V^{\dagger}$ ). Then the unitary operator $V$ can be expressed in terms of a Hermitian operator $h$ :

$$
V=\gamma_{5} h=\left(\begin{array}{cc}
\mathbb{I} & 0 \\
0 & -I
\end{array}\right)\left(\begin{array}{ll}
h_{1} & h_{2} \\
h_{2}^{\dagger} & h_{3}
\end{array}\right)=\left(\begin{array}{cc}
h_{1} & h_{2} \\
-h_{2}^{\dagger} & -h_{3}
\end{array}\right),
$$

where $h_{1}^{\dagger}=h_{1}$ and $h_{3}^{\dagger}=h_{3}$. Using the unitarity condition $V^{\dagger} V=\mathbb{I}$, we have $h^{2}=\mathbb{I}$ :

$$
h^{2}=\left(\begin{array}{cc}
h_{1}^{2}+h_{2} h_{2}^{\dagger} & h_{1} h_{2}+h_{2} h_{3} \\
h_{2}^{\dagger} h_{1}+h_{3} h_{2}^{\dagger} & h_{2}^{\dagger} h_{2}+h_{3}^{2}
\end{array}\right)=\left(\begin{array}{cc}
\text { I } & 0 \\
0 & I
\end{array}\right) .
$$

Then we immediately obtain

$$
D_{c}=(\mathbb{I}+V)(\mathbb{I}-V)^{-1}=\left[\begin{array}{cc}
0 & \left(\mathbb{I}-h_{1}\right)^{-1} h_{2} \\
-h_{2}^{\dagger}\left(\mathbb{I}-h_{1}\right)^{-1} & 0
\end{array}\right]
$$

and

$$
D_{c}^{-1}=(\mathbb{I}-V)(I+V)^{-1}=\left[\begin{array}{cc}
0 & -\left(\mathbb{I}+h_{1}\right)^{-1} h_{2} \\
h_{2}^{\dagger}\left(I+h_{1}\right)^{-1} & 0
\end{array}\right] .
$$

The last formula together with Eq. (6) reduces the task of computing $D^{-1}$ for any $R$ to that of computing $\left(\mathrm{I}+h_{1}\right)^{-1}$ only. For a four-dimensional lattice of volume $\Omega=L^{4}$, the former is to compute the inverse of a complex matrix of size $4 L^{4} \times 4 L^{4}$ (multiplicities due to the color and the flavor have been suppressed), while the latter only amounts to computing the inverse of a Hermitian matrix of size $2 L^{4} \times 2 L^{4}$. Further simplifications are possible, however, depending on the actual form of $V\left(D_{c}\right)$ as well as the algorithm used for computing the inverse. We do not intend to go into these technical details in this paper. In principle, formula (12) provides the proper way to compute the fermion propagator for any Ginsparg-Wilson fermion and serves as a starting point for devising highly optimized algorithms for computing the fermion propagators.

In order to investigate the spontaneous chiral symmetry breaking $(\chi \mathrm{SB})$ of $\mathrm{QCD}$, we usually introduce a mass parameter $m$ to the Dirac operator $D$, and then measure the chiral condensate in the thermodynamic limit $\Omega \rightarrow \infty$ followed by the massless limit $m \rightarrow 0$. The proper way to introduce the mass parameter to the Dirac operator $D$ is to add the mass $m$ to the chirally symmetric $D_{c}$ as in the continuum theory, but not to $D$ directly. Then the GW-Dirac operator (1) becomes

$$
D=\left(D_{c}+m\right)\left(\mathbb{I}+R D_{c}\right)^{-1} .
$$

It should be noted that the mass $m$ is not added to the $D_{c}$ in the last inverse operator which is inserted for breaking the chiral symmetry in the manner of Ginsparg and Wilson. Then the fermion propagator for massive GW fermions is

$$
D^{-1}=R+(I-m R)\left(D_{c}+m\right)^{-1},
$$

where

$$
\left(D_{c}+m\right)^{-1}=\left(\begin{array}{cc}
m\left(\mathbb{I}-h_{1}\right) B & -B h_{2} \\
h_{2}^{\dagger} B & m^{-1}\left[I-h_{2}^{\dagger} B\left(\mathbb{I}-h_{1}\right)^{-1} h_{2}\right]
\end{array}\right)
$$

and

$$
B=\left[\mathbb{I}+m^{2}+\left(\mathbb{I}-m^{2}\right) h_{1}\right]^{-1} .
$$

Again, the amount of computations using above formulas is significantly less than that of computing $\left(D_{c}+m\right)^{-1}$ directly. Once $\left(D_{c}+m\right)^{-1}$ is evaluated, then it takes almost no time to obtain $D^{-1}$ for any $R$ using Eq. (14).

Now we can write down the usual expression for the chiral condensate:

$$
\chi=\lim _{m \rightarrow 0} \lim _{\Omega \rightarrow \infty} \frac{1}{\Omega}\left\langle\sum_{x} \operatorname{tr}\left[\left.D^{-1}(x, x)\right|_{R=0}\right]\right\rangle,
$$

where the brackets $\langle\cdots\rangle$ denote averaging over each gauge configuration with the weight $\operatorname{det}(D) \exp \left(-A_{g}\right)$, and $A_{g}$ is the pure gauge action. The reason to set $R=0$ in $D^{-1}$ is obvious, since it is an arbitrary operator introduced for breaking the chiral symmetry in the manner of Ginsparg and Wilson; thus it is irrelevant to the physical $\chi \mathrm{SB}$ of $\mathrm{QCD}$, and must be dropped. ${ }^{1}$ Putting Eq. (14) into Eq. (17), we have

$$
\chi=\lim _{m \rightarrow 0} \lim _{\Omega \rightarrow \infty} \frac{1}{\Omega}\left\langle\sum_{x} \operatorname{tr}\left[\left(D_{c}+m\right)^{-1}(x, x)\right]\right\rangle .
$$

The subtleness of Eq. (18) lies in its two limiting processes. If the order of these two limiting processes is exchanged, then $\chi$ must be zero since spontaneous chiral symmetry breaking could not occur on a finite lattice for exactly massless fermions. Using Eq. (15), we can simplify Eq. (18) to the following expression:

$$
\chi=\lim _{m \rightarrow 0} \lim _{\Omega \rightarrow \infty} \frac{1}{\Omega}\left\langle\sum_{x} 2 m \operatorname{tr}\left[\left(\mathrm{I}-h_{1}\right) B\right](x, x)\right\rangle,
$$

where $B$ is defined in Eq. (16). This is a simpler expression than Eq. (18) since it only involves $h_{1}$, the Hermitian submatrix of $h=\gamma_{5} V$ defined in Eq. (9). The most time consuming operation in Eq. (19) is to evaluate the inverse matrix $B$.

Now we restore the weight $\operatorname{det}(D) \exp \left(-A_{g}\right)$ embedded inside the brackets $\langle\cdots\rangle$ and rewrite Eq. (19) as the following:

$$
\begin{aligned}
\chi= & \lim _{m \rightarrow 0} \lim _{\Omega \rightarrow \infty} \frac{1}{\Omega} \frac{1}{Z} \int[d U] \exp \left(-A_{g}\right) \operatorname{det}(D) \\
& \times \sum_{x} 2 m \operatorname{tr}\left[\left(I-h_{1}\right) B\right](x, x),
\end{aligned}
$$

\footnotetext{
${ }^{1}$ Note that setting $R=0$ in Eq. (17) does not necessarily reinstall doubling if $D_{c}$ is nonlocal, as has been discussed in detail in Ref. [4].
} 
where

$$
Z=\int[d U] \exp \left(-A_{g}\right) \operatorname{det}(D)
$$

and

$$
\operatorname{det}(D)=\operatorname{det}\left(D_{c}\right) \operatorname{det}\left(\mathbb{I}+R D_{c}\right)^{-1},
$$

using Eq. (1). From the GW relation, Eq. (2), it is obvious that we can redefine $D^{\prime}=R D$ such that $D^{\prime}$ satisfies Eq. (2) with $R=1$. However, one cannot set $R=1$ to the valence quarks since that would produce terms which are not relevent to the physical chiral symmetry breaking in QCD, as discussed after Eq. (17). On the other hand, for quarks in the internal quark loops, the chiral symmetry must be broken such that $D$ can be local and free of species doubling; then the fermionic determinant and the axial anomaly can turn out to be correct. Therefore we can set $R$ to 1 in Eq. (22), provided that $D_{c}$ has the correct classical continuum limit. Using Eq. (11), we obtain

$$
\operatorname{det}(D)=\operatorname{det}\left(\frac{I+h_{1}}{2}\right)=\operatorname{det}\left(\frac{I-h_{3}}{2}\right)
$$

In topologically nontrivial gauge sectors, the zero modes of $D$ correspond to the -1 eigenmodes of $h_{1}$, but the +1 eigenmodes of $h_{3}$. Further implementations of computing $\chi$ depend on the actual form of $V$ (or $D_{c}$ ) as well as the simulation algorithms. However, we will not go into these technical details in this paper. Now we put the results together and rewrite the chiral condensate in the following formula:

$$
\begin{aligned}
\chi= & \lim _{m \rightarrow 0} \lim _{\Omega \rightarrow \infty} \frac{1}{\Omega} \frac{1}{Z} \int[d U] \exp \left(-A_{g}\right) \operatorname{det}\left(\frac{\mathbb{I}+h_{1}}{2}\right) \\
& \times \sum_{x} 2 m \operatorname{tr}\left[\left(\mathbb{I}-h_{1}\right)\left[\mathbb{I}+m^{2}+\left(\mathbb{I}-m^{2}\right) h_{1}\right]^{-1}(x, x)\right],
\end{aligned}
$$

where $h_{1}$ is the Hermitian submatrix defined in Eq. (9) and

$$
Z=\int[d U] \exp \left(-A_{g}\right) \operatorname{det}\left(\frac{\mathbb{I}+h_{1}}{2}\right)
$$

If the chirally symmetric $D_{c}$ is initially specified as

$$
D_{c}=\left(\begin{array}{cc}
0 & -D_{L}^{\dagger} \\
D_{L} & 0
\end{array}\right)
$$

but not in terms of $V=\gamma_{5} h$, then it is straightforward to rewrite all formulas for the fermion propagators and the chiral condensate in terms of $D_{L}$.

Finally, we note that Neuberger [8] recently obtained an expression for the chiral condensate in the massless limit and in a fixed gauge background, in the framework of the overlap formalism [9]. Neuberger's result would agree with our general formula (24) if the mass parameter is introduced into the fermion propagator and the formula (15) is used.

In summary, we have derived analytic formulas for the $\mathrm{GW}$ fermion propagators for the massless and the massive cases, respectively. These formulas provide the proper way to compute $\mathrm{GW}$ fermion propagators in analytic studies as well as numerical ones. They also serve as a starting point for devising optimized algorithms for computing fermion propagators. An analytic formula for the chiral condensate is derived and is ready for numerical implementation to Monte Carlo simulations including dynamical fermions.

This work was supported by the National Science Council, R.O.C., under the grant NSC88-2112-M002-016.
[1] P. Ginsparg and K. Wilson, Phys. Rev. D 25, 2649 (1982).

[2] P. Hasenfratz, Nucl. Phys. B525, 401 (1998).

[3] T.W. Chiu and S.V. Zenkin, Phys. Rev. D 59, 074501 (1999).

[4] T.W. Chiu, Phys. Lett. B 445, 371 (1999).

[5] T.W. Chiu, “'Topological phases in Neuberger-Dirac operator," hep-lat/9810002.
[6] M. Lüscher, Phys. Lett. B 428, 342 (1998).

[7] H.B. Nielsen and N. Ninomiya, Nucl. Phys. B185, 20 (1981); B193, 173 (1981).

[8] H. Neuberger, 'Lattice Chirality,', hep-lat/9807009.

[9] R. Narayanan and H. Neuberger, Nucl. Phys. B443, 305 (1995). 